\title{
Arterial Stiffening and Clinical Outcomes in Dialysis Patients
}

\author{
Akihiko Kato \\ Blood Purification Unit, Hamamatsu University Hospital, Hamamatsu, Japan
}

\section{Key Words}

Brachial-ankle pulse wave velocity $\cdot$ Cardio-ankle vascular index $\cdot$ Mortality $\cdot$ Sarcopenic obesity · Cognitive function · Hemodialysis

\begin{abstract}
Cardiovascular disease (CVD) is an important cause of morbidity and mortality in dialysis patients. Brachial-ankle pulse wave velocity (baPWV) is more efficient to handily assess arteriosclerosis than aortic PWV. The cardio-ankle vascular index (CAVI) is also a novel blood pressure-independent arterial stiffness parameter. In dialysis patients, both baPWV and CAVI are increased compared to general subjects. Several studies have demonstrated that increased baPWV is associated with carotid atherosclerosis and diastolic left ventricular dysfunction in hemodialysis (HD) patients. In addition, higher baPWV is related to all-cause and cardiovascular (CV) mortality. CAVI is similarly associated with CVD. However, baPWV is superior to CAVI as a predictor of CV outcomes in HD patients. Besides these outcomes, a close relationship exists between sarcopenia, abdominal visceral obesity and arterial stiffening. Reduction of thigh muscle mass is inversely correlated with baPWV and CAVI in males. Abdominal fatness is also associated with increased arterial stiffness in females. These observations provide further evidence of higher risk of CV events in HD patients with sarcopenic obesity. In addition, arterial stiffness is associated with cerebral small vessel disease and decreased cognitive function in the elderly. However, it is unknown whether arterial stiffness may be useful as an early indicator of cognitive decline in dialysis patients. Because dialysis patients are at risk of developing dementia, more studies are needed to elucidate the causal link between arterial stiffness and cognitive impairment.

(c) 2015 S. Karger AG, Basel
\end{abstract}


Kato: Arterial Stiffening and Clinical Outcomes in Dialysis Patients

\section{Introduction}

Arterial stiffness is based on structural alterations occurring before plaque or thrombosis formation in muscular and elastic vessels and is used as a surrogate marker of arteriosclerosis. There are several methods to assess pulse wave velocity (PWV); the most frequent methods are carotid-femoral PWV (cfPWV) and brachial-ankle PWV (baPWV). However, baPWV is time-efficient and technically simple; thus, measurement of baPWV is currently prevalent especially in eastern Asian countries.

The cardio-ankle vascular index (CAVI) is a novel index which measures the overall stiffness of the artery from the origin of the aorta to the ankle according to the theory of stiffness parameter $\beta$. CAVI is less dependent on blood pressure at the time of measurement than PWV. CAVI also reflects smooth muscle cell contracture, so it might be useful in evaluating the real effects of blood pressure control on the muscular arteries [1].

In this review, we focus on the impact of arterial stiffening on clinical outcomes in dialysis patients. Bedsides well-known outcomes such as cardiovascular (CV) events and mortality, we discuss a plausible association of arterial stiffness with changes of body composition, such as sarcopenia and abdominal adiposity, and impaired cognitive function.

\section{Arterial Stiffening and Mortality}

\section{baPWV}

In the general population, increased baPWV is an independent predictor of mortality. There was a 6.8-fold higher risk of all-cause mortality in subjects with baPWV $>17.0 \mathrm{~m} / \mathrm{s}$ when compared to those with baPWV $<14.0 \mathrm{~m} / \mathrm{s}$ [2]. Similarly, baPWV $>17.0 \mathrm{~m} / \mathrm{s}$ is associated with poorer survival prognosis in diabetic patients with coronary artery disease (CAD) [3]. In hospitalized patients with acute ischemic stroke, those with higher baPWV ( $>22.63$ $\mathrm{m} / \mathrm{s}$ ) are at an increased risk for both all-cause and CV death compared to those with baPWV $<17.79 \mathrm{~m} / \mathrm{s}$ [4].

In patients with chronic kidney disease (CKD) stages G3-G5, baPWV decreases with a decline in the glomerular filtration rate [5]. Increased arterial stiffening causes a greater transmission of systolic blood pressure to the glomerular capillaries, thereby leading to an exacerbation of glomerular hypertension, a main determinant of progressive kidney damage. baPWV $>19.4 \mathrm{~m} / \mathrm{s}$ is also independently associated with progression to commencing dialysis or death [5].

Kitahara et al. [6] first demonstrated that baPWV $>23.8 \mathrm{~m} / \mathrm{s}$ was independently associated with all-cause mortality in patients on chronic hemodialysis (HD). They also found that increased baPWV was an independent predictor of all-cause and CV mortality in patients with an ankle-brachial index (ABI) of $\geq 0.9$ [6]. Higher baPWV ( $\geq 18.0 \mathrm{~m} / \mathrm{s})$ was also associated with cardiovascular mortality in patients with a normal ABI [7]. However, in HD patients, ABI is more useful than baPWV in predicting CV morbidity and mortality [8-10].

CAVI

Few studies examined whether CAVI can predict survival prognosis. We previously conducted a 39-month observational study and found that CAVI was not associated with total mortality in HD patients [9]. In addition, baPWV is superior to CAVI in predicting all-cause and CV death in HD patients aged 55-70 years with a normal ABI [11]. 
Kato: Arterial Stiffening and Clinical Outcomes in Dialysis Patients

Table 1. Summary of arterial stiffness and clinical outcomes in HD patients

\begin{tabular}{|c|c|c|c|c|c|}
\hline Patients, n & $\begin{array}{l}\text { Age, } \\
\text { years }\end{array}$ & Time on HD & $\begin{array}{l}\text { Cutoff, } \\
\mathrm{m} / \mathrm{s}\end{array}$ & Clinical outcomes & Ref. \\
\hline \multicolumn{6}{|l|}{ baPWV } \\
\hline 785 & $60 \pm 12$ & $6.5 \pm 5.8$ years & $\geq 23.8$ & total mortality & 6 \\
\hline 199 & $61 \pm 13$ & $132 \pm 84$ months & $\geq 18.0$ & total mortality & 7 \\
\hline 135 & $60 \pm 11$ & $110 \pm 93$ months & $\geq 16.6$ & CV mortality & 11 \\
\hline 68 & $60 \pm 2$ & $4.4(1-26)$ years & ND & maximal carotid IMT & 18 \\
\hline 142 & $64 \pm 11$ & $7.7 \pm 7.0$ years & ND & diastolic left ventricular dysfunction & 19 \\
\hline 160 & $59 \pm 13$ & $3,138 \pm 2,498$ days & 15.6 & maximal carotid IMT & 28 \\
\hline \multicolumn{6}{|l|}{$\overline{C A V I}$} \\
\hline 58 & $60 \pm 13$ & ND & $\geq 7.55$ & presence of CVD & 24 \\
\hline 103 & $65 \pm 11$ & $8.2 \pm 8.3$ years & $\geq 9.9$ & $\begin{array}{l}\text { fibrous tissue area at the brachial } \\
\text { arterial wall (\%) }\end{array}$ & 25 \\
\hline 160 & $59 \pm 13$ & $3,138 \pm 2,498$ days & $\geq 9.5$ & maximal carotid IMT & 28 \\
\hline
\end{tabular}

Values are presented as means \pm SD unless otherwise indicated. ND $=$ Not described.

\section{Arterial Stiffening and CV Events}

\section{$b a P W V$}

baPWV exhibits a close association with the classical Framingham risk score similar to cfPWV, the golden index of arterial stiffness [12]. baPWV is comparable to cfPWV in predicting the presence of stroke and CAD in the general population [12]. Higher baPWV is an independent predictor of CV events occurring after hospitalization $(\geq 17 \mathrm{~m} / \mathrm{s})$ and of a major CV event ( $\geq 18 \mathrm{~m} / \mathrm{s})$ in patients with acute coronary syndrome [13]. Increased baPWV $(>18$ $\mathrm{m} / \mathrm{s}$ ) is also associated with an incident CV event in the general subjects without a history of cardiovascular disease (CVD) [14]. A large-scale cohort provided evidence that baPWVs of 14 and $24 \mathrm{~m} / \mathrm{s}$ are statistically adequate cutoff points for CV events and mortality in patients with type 2 diabetes mellitus (DM), excluding those with an ABI of $<0.9$ [15]. baPWV ( $>22.25$ $\mathrm{m} / \mathrm{s}$ ) has an independent prognostic value for predicting functional outcome assessed by the modified Rankin Scale score 3 months after first-ever acute cerebral infarction [16].

In predialysis CKD patients, baPWV is associated with the severity of stenosis at carotid and intracranial arteries [17]. There is also a positive relationship between higher baPWV and more advanced carotid arteriosclerosis [18] and left ventricular dysfunction [19] in HD patients. However, baPWV is not useful for stratifying the risk of CV morbidity such as cardiac, cerebrovascular, and peripheral artery events during the 5-year follow-up in chronic HD patients [8].

\section{CAVI}

CAVI is elevated in persons who are at a high risk for CVD [20]. CAVI correlates positively with maximal intima-media thickness (IMT) of the common carotid artery in patients undergoing coronary angiography (CAG) [21]. CAVI is also positively correlated with coronary artery calcification in DM patients [22]. A persisting increment of CAVI is also an independent risk factor of future CVD events in patients with CAD [23]. In HD patients, CAVI >7.55 is predictable of CVD with both a sensitivity and specificity of 0.79 [24]. CAVI is also more predictable of the percentile of histological fibrous area at the brachial artery wall than baPWV [25].

The associations of baPWV and CAVI with mortality, morbidity and arteriosclerotic changes are summarized in table 1. 


\section{Comparison between baPWV and CAVI}

In patients undergoing CAG, CAVI is more closely associated with carotid IMT and the presence of multiple vessel disease than baPWV [26]. CAVI, but not baPWV, is also correlated with the parameters of left ventricular diastolic indices from echocardiography in patients with chest pain syndrome who have undergone CAG [27]. Taken together, CAVI may be more related to arteriosclerosis than baPWV in patients with CVD.

In HD patients, both baPWV and CAVI have identical power to predict maximal carotid IMT. The CAVI value is increased just after a HD session when the fluid removal rate is $>5 \%$ of the dry weight, indicating that CAVI is easily influenced by excess fluid removal [28]. We also showed that baPWV $>16.6 \mathrm{~m} / \mathrm{s}$ was an independent predictor of CV events, while CAVI was not in HD patients with an ABI ranging from 0.9 to 1.3 [11]. It follows from these observations that baPWV may be superior to CAVI as a surrogate marker of arteriosclerosis in HD patients. The measurement of CAVI is adjusted by the equation of stiffness $\beta$, which is essentially applied to a portion of the aorta. Since CAVI is inversely correlated with the calcified area of the aortic wall in HD patients [29], advanced aortic calcification may attenuate smooth muscle contractility of the elastic aorta, thereby leading to underestimation of their values.

It is generally accepted that stiffness of the brachial artery is less affected by age than stiffness of the aorta. The physiological gradient of stiffness between the aorta and the peripheral artery is hypothesized to mitigate the transmission of forward travelling wave energy into the microcirculation, therefore providing protection against pulse pressureinduced changes in microvasculature. In HD patients, a reduction of brachial stiffness was inversely associated with basal aortic stiffness, indicating that a regression of brachial stiffness may be an adaptive response to an increased aortic stiffness [30]. Recently, aorticbrachial mismatch of arterial stiffness, assessed by the ratio of cfPWV to carotid-radial PWV, has been shown to be more associated with overall mortality than cfPWV in HD patients [31]. Because baPWV and CAVI assume that the arterial tree is homogeneous, further studies are needed to evaluate PWV based on the exploration of the heterogeneity of the arterial tree.

\section{Arterial Stiffening and Body Composition}

Recently, changes of regional body composition, especially reduced thigh muscle mass and increased abdominal fatness, have been shown to be closely related to arteriosclerotic changes in the general and dialysis populations.

\section{Decreased Muscle Mass Volume}

In patients with hemiparesis, baPWV is greater on paretic legs than on nonparetic legs in association with loss of muscle mass [32]. On computed tomography (CT), a mid-thigh muscle area was negatively correlated with baPWV in male subjects $[33,34]$. The $\mathrm{ABC}$ study also showed that higher PWV is associated with a more profound decline of the sarcopenic index in older men aged 70-79 years [35]. CAVI is also related to the skeletal muscle mass index in community-dwelling older adults [36]. Similarly, the thigh muscle mass area is reported to be positively correlated with baPWV and CAVI in HD patients [37].

\section{Increased Abdominal Fatness}

The abdominal visceral fat mass is positively related to cfPWV in healthy subjects [38]. The ratio of appendicular skeletal muscle mass index to visceral fat area, a novel index of sarcopenic obesity, is an independent predictor of baPWV [39]. cfPWV was also associated positively with body fat mass volume and negatively with fat-free mass volume at the extremities of Asian Indian patients with type 2 DM [40]. 


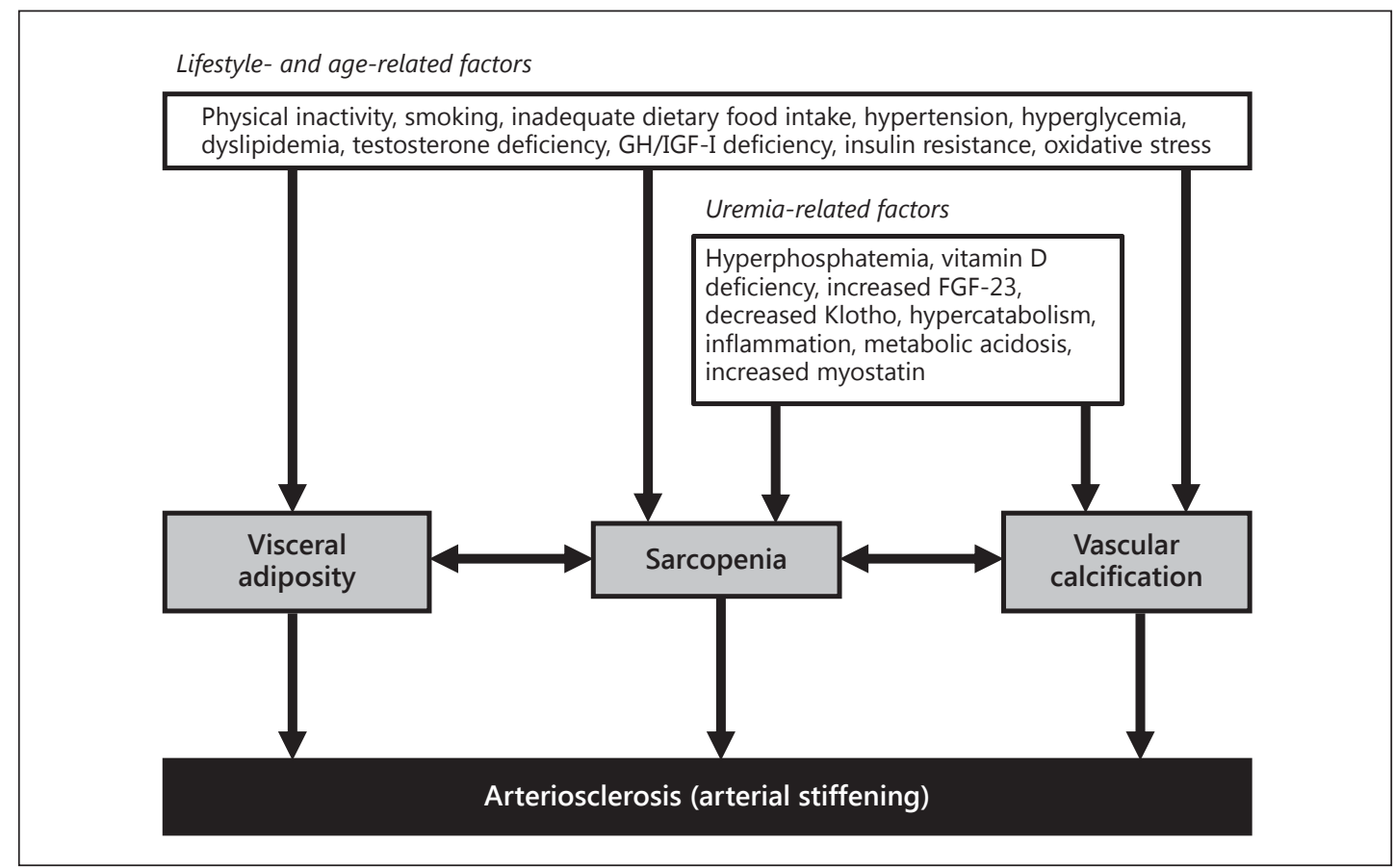

Fig. 1. Possible underlying mechanisms between sarcopenia, visceral obesity, vascular calcification and arteriosclerosis. Lifestyle- and age-related factors such as physical inactivity (sedentary life), smoking, inadequate dietary food intake, hypertension, hyperglycemia, dyslipidemia, deficiency of sex hormone (testosterone, estrogen), growth hormone (GH), and insulin-like growth factor-1, insulin resistance, and oxidative stress could lead to skeletal muscle mass loss (sarcopenia), abdominal adiposity, and vascular calcification, thereby resulting in an exacerbation of arteriosclerosis. In addition, the uremia-related factors such as vitamin D deficiency, hypercatabolism, chronic inflammation, metabolic acidosis, and increased myostatin expression result in skeletal muscle atrophy. CKD-related mineral and bone disorders such as hyperphosphatemia, increased fibroblast growth factor-23 and decreased arterial Klotho protein also accelerate vascular calcification, a major mediator of arteriosclerosis. Increased adipose tissue increases macrophage infiltration in the tissue and enhances production of proinflammatory cytokines such as tumor necrosis factor- $\alpha$ and interleukin-6, accompanying with dysregulated secretion of leptin and adiponectin. These adipokines can provoke adverse effects on skeletal muscle. Decreased skeletal muscle mass reduces basal energy expenditure and lowers muscular interleukin-15 production, which works to prevent the differentiation of adipocytes. In addition, sarcopenia and abdominal fatness also induce insulin resistance, and additively facilitate arteriosclerotic processes.

There is also a positive relationship between the abdominal visceral fat area and baPWV in diabetic HD patients [41]. An inverse association was found between abdominal visceral fatness and baPWV in female HD patients [42]. In peritoneal dialysis patients, fat mass volume measured by multiple frequency bioelectrical impedance analysis was also associated positively with cfPWV [43].

\section{Underlying Mechanisms between Arterial Stiffening and Changes of Body Composition}

There are plausible mechanisms that explain why muscle wasting and abdominal adiposity may be associated with arterial stiffness in HD patients (fig. 1). First, recent studies have suggested that both increased PWV [44] and thigh sarcopenia [45] are correlated with testosterone deficiency, especially in men. Because testosterone is a vasoactive hormone that predominantly has vasodilatory action on vascular beds [46], and it enhances 
Kato: Arterial Stiffening and Clinical Outcomes in Dialysis Patients

degradation of elastin matrix proteins via increased matrix metalloproteinase- 2 in arterial smooth muscle cells [47], low testosterone may facilitate atherogenesis and skeletal muscle wasting.

Second, skeletal muscle is the main site of insulin-mediated glucose disposal, so reduced muscle mass with increased visceral fat may result in insulin resistance, thereby accelerating systemic arteriosclerosis. We previously showed that the ratio of high molecular weight to total adiponectin, a better predictor for insulin resistance, is positively correlated with both baPWV and CAVI in HD patients [29].

Third, both sarcopenia and abdominal adiposity are well correlated with proinflammatory status [40,48]. Because serum C-reactive protein is an independent risk factor for baPWV in dialysis patients [49], increased arterial stiffening may reflect the presence of an inflammatory reaction at arteries in the whole body.

Finally, low physical activity is associated with increased baPWV in middle-aged to elderly men [34]. Sedentary life is very common in HD patients and links to muscle atrophy and visceral fatness. Recently, a randomized controlled study [50] has demonstrated that moderate-intensity resistance exercise training ( 3 days per week) for a 12-month period concomitantly improves cfPWV, cardiopulmonary fitness and abdominal adiposity in patients with CKD stages G3-G4.

Possible underlying mechanisms between sarcopenia, visceral adiposity, vascular calcification and arteriosclerosis are presented in figure 1.

\section{Arterial Stiffening and Cognitive Function}

Arterial stiffness appears to play a major role in the relationship between hypertension and its consequence in the brain, including cognitive impairment and the pathological features of Alzheimer's disease. Aortic stiffness loses its cushioning capacity, and thus pulsatile pressure is easily transmitted to the brain vessels. Increased pulse pressure in the brain can provoke structural changes and dysfunction of its microcirculation, resulting in damage to small cerebral vessels. This, in turn, leads to cerebral hypoperfusion, thereby causing brain atrophy particularly in the areas of the frontal-subcortical brain that specifically control executive and motor function, and thus impairing cognitive domains [51].

Recently, $\beta$-amyloid plaque deposition in the brain on positron emission topography imaging has been shown to be more strongly associated with baPWV than cfPWV in elderly adults aged 83-96 years [52]. A systematic review revealed that increased baPWV or cfPWV is associated with radiological findings of cerebral small vessel disease, such as white matter hyperintensity or lacunar infarction, and decreased cognitive function in general subjects [53]. CAVI was also independently associated with cerebral small vessel disease in healthy young and middle-aged subjects [54]. A high CAVI value is predictive of cognitive decline over 4 years in community-dwelling elderly people [55].

In patients with end-stage kidney disease, baPWV was associated with the spot number of silent lacunar infarction on brain MRI [17]. However, the link between arterial stiffening and cognitive decline and dementia remains to be clarified in dialysis patients.

\section{Summary}

Besides CV morbidity and mortality, there is evidence that arterial stiffening is related to sarcopenia and abdominal visceral fatness in HD patients. Reduction of thigh muscle mass is inversely correlated with arterial stiffness in male patients. Abdominal fatness is negatively 
associated with arterial stiffness in female patients. These findings provide further evidence of a higher risk of CV events in sarcopenic obesity. In addition, arterial stiffness is associated with cerebral small vessel disease and decreased cognitive function. Since an association of arterial stiffness with cognitive decline is not elucidated in the dialysis population, further studies are needed to understand whether arterial stiffness has a causal effect on cognitive decline and dementia, and if so, through what mechanisms.

\section{Disclosure Statement}

The author has no conflicts of interest to declare.

\section{References}

1 Shirai K, Utino J, Saiki A, et al: Evaluation of blood pressure control using a new arterial stiffness parameter, cardio-ankle vascular index (CAVI). Curr Hypertens Rev 2013;9:66-75.

$>2$ Turin TC, Kita Y, Rumana N, et al: Brachial-ankle pulse wave velocity predicts all-cause mortality in the general population: findings from the Takashima study, Japan. Hypertens Res 2010;33:922-925.

-3 Nakamura M, Yamashita T, Yajima J, et al: Brachial-ankle pulse velocity as a risk stratification index for the short-term prognosis of type 2 diabetic patients with coronary artery disease. Hypertens Res 2010;33:10181024.

4 Kim J, Song TJ, Song D, et al: Brachial-ankle pulse wave velocity is a strong predictor for mortality in patients with acute stroke. Hypertension 2014;64:240-246.

5 Chen SC, Chang JM, Liu WC, et al: Brachial-ankle pulse wave velocity and rate of renal function decline and mortality in chronic kidney disease. Clin J Am Soc Nephrol 2011;6:724-732.

6 Kitahara T, Ono K, Tsuchida A, et al: Impact of brachial-ankle pulse wave velocity and ankle-brachial blood pressure index on mortality in hemodialysis patients. Am J Kidney Dis 2005;46:688-696.

7 Morimoto S, Yurugi T, Aota Y, et al: Prognostic significance of ankle-brachial index, brachial-ankle pulse wave velocity, flow-mediated dilation, and nitroglycerin-mediated dilation in end-stage renal disease. Am J Nephrol 2009;30:55-63.

8 Tanaka M, Ishii H, Aoyama T, et al: Ankle brachial pressure index but not brachial-ankle pulse wave velocity is a strong predictor of systematic atherosclerotic morbidity and mortality in patients on maintenance hemodialysis. Atherosclerosis 2011;219:643-647.

-9 Kato A, Takita T, Furuhashi M, et al: A small reduction in the ankle-brachial index is associated with increased mortality in patients on chronic hemodialysis. Nephron Clin Pract 2010;114:c29-c37

10 Kuwahara M, Hasumi S, Mandai S, et al: Rate of ankle-brachial index decline predicts cardiovascular mortality in hemodialysis patients. Ther Apher Dial 2014;18:9-18.

11 Kato A, Takita T, Furuhashi M, et al: Brachial-ankle pulse wave velocity and the cardio-ankle vascular index as a predictor of cardiovascular outcomes in patients on regular hemodialysis. Ther Apher Dial 2012;16:232241.

12 Tanaka H, Munakata M, Kawano Y, et al: Comparison between carotid-femoral and brachial-ankle pulse wave velocity as measures of arterial stiffness. J Hypertens 2009;27:2022-2027.

13 Tomiyama H, Koji Y, Yambe M, et al: Brachial-ankle pulse wave velocity is a simple and independent predictor of prognosis in patients with acute coronary syndrome. Circ J 2005;69:815-822.

14 Takashima N, Turin TC, Matusi K, et al: The relationship of brachial-ankle pulse wave velocity to future cardiovascular disease events in the general Japanese population: the Takashima study. J Hum Hypertens 2014;28: 323-327.

15 Maeda Y, Inoguchi T, Etoh E, et al: Brachial-ankle pulse wave velocity predicts all-cause mortality and cardiovascular events in patients with diabetes: the Kyushu Prevention Study of Atherosclerosis. Diabetes Care 2014;37:2383-2390.

16 Kim J, Song TJ, Kim EH, et al: Brachial-ankle pulse wave velocity for predicting functional outcome in acute stroke. Stroke 2014;45:2305-2310.

17 Washida N, Wakino S, Hayashi K, et al: Brachial-ankle pulse wave velocity predicts silent cerebrovascular disease in patients with end-stage renal disease. J Atheroscler Thromb 201;17:165-172.

$\$ 18$ Munakata M, Sakuraba J, Tayama J, et al: Higher brachial-ankle pulse wave velocity is associated with more advanced carotid atherosclerosis in end-stage renal disease. Hypertens Res 2005;28:9-14.

19 Fujiu A, Ogawa T, Matsuda N, et al: Aortic arch calcification and arterial stiffness are independent factors for diastolic left ventricular dysfunction on chronic hemodialysis patients. Circ J 2008;72:1768-1772. 
20 Namekata T, Suzuki K, Ishizuka N, et al: Establishing baseline criteria of cardio-ankle vascular index as anew indicator of arteriosclerosis: a cross-sectional study. BMC Cardiovasc Disord 2011;11:51.

21 Nakamura K, Tomaru T, Yamamura S, et al: Cardio-ankle vascular index is a candidate predictor of coronary atherosclerosis. Circ J 2008;72:598-604.

22 Mineoka Y, Fukui M, Tanaka M, et al: Relationship between cardio-ankle vascular index (CAVI) and coronary artery calcification (CAC) in patients with type 2 diabetes mellitus. Heart Vessel 2012;27:160-165.

-23 Otsuka K, Fukuda S, Shimada K, et al: Serial assessment of arterial stiffness by cardio-ankle vascular index for prediction of future cardiovascular events in patients with coronary artery disease. Hypertens Res 2014;37: 1014-1020.

24 Takenaka T, Hoshi H, Kato N, et al: Cardio-ankle vascular index to screen cardiovascular disease in patients with end-stage renal diseases. J Atheroscler Thromb 2008;15:339-344.

25 Ichihara A, Yamashita N, Takemitsu T, et al: Cardio-ankle vascular index and ankle pulse wave velocity as a marker of arterial fibrosis in kidney failure treated by hemodialysis. Am J Kidney Dis 2008;52:947-955.

-26 Izuhara M, Shioji K, Kadota S, et al: Relationship of cardio-ankle vascular index (CAVI) to carotid and coronary arteriosclerosis. Circ J 2008;72:1762-1767.

27 Takaki A, Ogawa H, Wakayama T, et al: Cardio-ankle vascular index is superior to brachial-ankle pulse wave velocity as an index of arterial stiffness. Hypertens Res 2008;31:1347-1355.

28 Ueyama K, Miyata M, Kubozono T, et al: Noninvasive indices of arterial stiffness in hemodialysis patients. Hypertens Res 2008;32:716-720.

29 Kato A, Odamaki M, Ishida J, et al: Association of high-molecular-weight to total adiponectin ratio with pulse wave velocity in hemodialysis patients. Nephron Clin Pract 2008;109:c18-c24.

30 Utescu MS, Couture V, Mac-Way F, et al: Determinants of progression of aortic stiffness in hemodialysis patients: a prospective longitudinal study. Hypertension 2013;62:154-160.

-31 Fortoer C, Mac-Way F, Desmeules S, et al: Aortic brachial stiffness mismatch and mortality in dialysis population. Hypertension 2015;65:378-384.

32 Okabe R, Inaba M, Sakai S, et al: Increased arterial stiffening and thickening in the paretic lower limb in patients with hemiparesis. Clin Sci (London) 2004;106:613-618.

-33 Kohara K, Ochi M, Tabara Y, et al: Arterial stiffness in sarcopenic visceral obesity in the elderly: J-SHIPP study. Int J Cardiol 2012;158:146-148.

34 Ochi M, Kohara K, Tabara Y, et al: Arterial stiffness is associated with low thigh muscle mass in middle-aged to elderly men. Atherosclerosis 2010;212:327-332.

35 Abbatecola AM, Chiodini P, Gallo C, et al: Pulse wave velocity is associated with muscle mass decline: Health ABC study. Age (Dordr) 2012;34:469-478.

-36 Aurélio RA, Sampaio C, Sampaio PYSW, et al: Arterial stiffness is associated with low skeletal muscle mass in Japanese community-dwelling older patients. Geriatr Gerontol Int 2014;14(suppl 1):109-114.

-37 Kato A, Ishida J, Endo Y, et al: Association of abdominal visceral adiposity and thigh sarcopenia with changes of arteriosclerosis in haemodialysis patients. Nephrol Dial Transplant 2011;26:1967-1976.

-38 Lee Y, Shin H, Vassy JL, et al: Comparison of regional body composition and its relation with cardiometabolic risk between BMI-matched young and old subjects. Atherosclerosis 2012;224:258-265.

39 Kim TN, Park MS, Lim KI, et al: Skeletal muscle mass to visceral fat area ratio is associated with metabolic syndrome and arterial stiffness: the Korean Sarcopenic Obesity Study (KSOS). Diabetes Res Clin Pract 2011; 93:285-291.

-40 Anoop S, Mistra A, Bhardwaj S, et al: High body fat and low muscle mass are associated with increased arterial stiffness in Asian Indians in North India. J Diabetes Complications 2015;20:38-43.

41 Yurugi T, Morimoto S, Okamoto T, et al: Accumulation of visceral fat in maintenance hemodialysis patients. Clin Exp Nephrol 2012;16:156-163.

42 Kato A, Takita T, Kumagai H: Relationship between arterial stiffening and skeletal muscle atrophy in hemodialysis patients: a gender comparative study. J Cachexia Sarcopenia Muscle 2014;5:247-249.

43 Lu Q, Cheng LT, Wang T, et al: Visceral fat, arterial stiffness, and endothelial function in peritoneal dialysis patients. J Ren Nutr 2008;18:495-502.

44 Kyriazis J, Tzanakis I, Stylianou K, et al: Low serum testosterone, arterial stiffness and mortality in male haemodialysis patients. Nephrol Dial Transplant 2011;26:2971-2977.

-45 Kojo G, Yoshida T, Ohkawa S, et al: Association of serum total testosterone concentration with skeletal muscle mass in men under hemodialysis. Int Urol Nephrol 2014;46:985-991.

46 Kelly DM, Jones TH: Testosterone: a vascular hormone in health and disease. J Endocrinol 2013;217:R47-R71.

47 Natoli AK, Medley TL, Ahimastos AA, et al: Sex steroids modulate human aortic smooth muscle cell matrix protein deposition and matrix metalloproteinase expression. Hypertension 2005;46:1129-1134.

$\checkmark 48$ Honda H, Qureshi AR, Axelsson J, et al: Obese sarcopenia in patients with end-stage renal disease is associated with inflammation and increased mortality. Am J Clin Nutr 2007;86:633-638.

49 Kuang DW, Li CL, Kuok U, et al: Risk factors associated with brachial-ankle pulse wave velocity among peritoneal dialysis patients in Macao. BMC Nephrol 2012;13:143.

50 Greenwood SA, Koufaki P, Mercer TH, et al: Effect of Exercise Training on Estimated GFR, vascular health, and cardiorespiratory fitness in patients with CKD: a pilot randomized controlled trial. Am J Kidney Dis 2015;65: 425-434. 
Kato: Arterial Stiffening and Clinical Outcomes in Dialysis Patients

51 Al Hazzouri AZ, Yaffe K: Arterial stiffness and cognitive function in the elderly. J Alzheimers Dis 2014;42:S503S514.

52 Hughes TM, Kuller LH, Barinas-Mitchell EJM, et al: Pulse wave velocity is associated with $\beta$-amyloid deposition in the brains of very elderly adults. Neurology 2013;81:1711-1718.

53 Singer J, Trollor JN, Baune BT, et al: Arterial stiffness, the brain and cognition: a systematic review. Ageing Res Rev 2014;15:16-27.

54 Choi SY, Park HE, Seo H, et al: Arterial stiffness using cardio-ankle vascular index reflects cerebral small vessel disease in healthy young and middle aged subjects. J Atheroscler Thromb 2013;20:178-185.

55 Yamamoto N, Yamanaka G, Ishikawa M, et al: Cardio-ankle vascular index as a predictor of cognitive impairment in community-dwelling elderly people: four-year follow-up. Dement Geriatr Cogn Disord 2009;28:153-158. 